\title{
The Relationship between Aging and Photic Driving EEG Response
}

\author{
Tadanori FUKAMI $^{\dagger a}$, Takamasa SHIMADA ${ }^{\dagger \dagger}$, Members, Fumito ISHIKAWA ${ }^{\dagger \dagger \dagger}$, Bunnoshin ISHIKAWA ${ }^{\dagger \dagger \dagger}$, \\ and Yoichi SAITO ${ }^{\dagger \dagger \dagger}$, Nonmembers
}

\begin{abstract}
SUMMARY The present study examined the evaluation of aging using the photic driving response, a measure used in routine EEG examinations. We examined 60 normal participants without EEG abnormalities, classified into three age groups (20 29, 30 59 and over 60 years; 20 participants per group). EEG was measured at rest and during photic stimulation (PS). We calculated Z-scores as a measure of enhancement and suppression due to visual stimulation at rest and during PS and tested for between-group and intraindividual differences. We examined responses in the alpha frequency and harmonic frequency ranges separately, because alpha suppression can affect harmonic frequency responses that overlap the alpha frequency band. We found a negative correlation between Z-scores for harmonics and age by fitting the data to a linear function (CC: -0.740$)$. In contrast, Z-scores and alpha frequency were positively correlated (CC: 0.590$)$.

key words: EEG, photic driving response, aging, Z-score
\end{abstract}

\section{Introduction}

This study was conducted to test whether the photic driving response, a routine electroencephalography (EEG) examination, can be used to assess the effects of biological aging on the brain. To this end, we examined the relationship between electrophysiological activity in different frequency ranges and ages. A detailed understanding of EEG correlates of aging in the brain could provide additional clinically useful information in EEG diagnosis.

Studies of the relationship between EEG and aging have established that the peak alpha frequency decreases with age. Lindsley examined the occipital alpha rhythm in healthy children, analyzing the frequency and amplitude of the EEG signal. However, there is little variation in peak frequency with aging, meaning that it does not provide a sensitive measure of the effects of aging in the brain [1]. In the current study, we used the photic driving response as a primary EEG measure. The photic driving response is measured in routine EEG examinations in clinical diagnosis by presenting subjects with photic stimulation (PS) and

\footnotetext{
Manuscript received April 4, 2011.

Manuscript revised May 20, 2011.

$\dagger$ The author is with the Department of Informatics, Faculty of Engineering, Yamagata University, Yonezawa-shi, 992-8510

${ }^{\dagger}$ The author is with the Faculty of Information and Environment, Tokyo Denki University, Inzai-shi, 270-1382 Japan.

${ }^{\dagger \dagger}$ The authors are with the Hotokukai Utsunomiya Hospital, Utsunomiya-shi, 320-8521 Japan.

${ }^{\dagger}+\dagger$ The author is with the Institute for EEG Analysis, Tokyo, 114-0015 Japan.

a)E-mail: fukami@yz.yamagata-u.ac.jp

DOI: 10.1587/transinf.E94.D.1839
} Japan. measuring the elicited rhythmic activity. The effect of intermittent PS on human EEG was first studied by Adrian and Matthews [2], and numerous studies have subsequently been conducted to measure and analyze photic driving responses in humans [3], [4]. However, few studies have examined the relation between photic driving responses and biological aging in humans. Celesia et al. reported that the latencies of the first major negative and first major positive deflection of the visual evoked response were significantly delayed ( $p<0.001)$ with advancing age. In addition, the critical frequency photic driving (CFPD) response (defined as the highest frequency of photic driving response expressed in flashes per second) exhibited an inverse correlation with age, decreasing in older subjects [5]. However, the study did not examine the frequency components of the photic driving response, focusing solely on its amplitude/latency and critical frequency. However, amplitude and latency are susceptible to the effects of EEG signal averaging for estimation.

In addition to obtaining the relationship between aging and photic driving response, our method might be used to remove bias due to aging in EEG evaluations.

\section{Methods}

Multichannel EEG was recorded using a Nihon Koden polygraph (EEG-1100) with a $0.3 \mathrm{~s}$ time constant, $60 \mathrm{~Hz}$ high cut filter, and a $97.5 \mathrm{nV}$ quantization level. EEG signals were measured from 19 electrodes placed according to the international 10/20 system with monopolar derivation from bilateral reference electrodes attached to the corresponding earlobes.

A cycle involving a block without PS (at rest) for $10 \mathrm{~s}$ followed by a block with PS for $10 \mathrm{~s}$ was repeated. Six PS frequencies were used (3, 6, 7, 10, 14 and $18 \mathrm{~Hz})$, and participants were recorded during a state of relaxed wakefulness with closed eyes. EEG signals were digitized at a sampling frequency of $200 \mathrm{~Hz}$. The photic pulse stimuli had the following parameters: the brightness of the flash stimulus was $30,000 \mathrm{~cd} / \mathrm{m}^{2}$, the light intensity was $4.01 \mathrm{x} / \mathrm{s}$, the pulse width was $2 \mathrm{~ms}$, and the equipment was placed $30 \mathrm{~cm}$ in front of the subjects. All data reported in this study were recorded from participants at Utsunomiya Hospital after obtaining informed consent. We measured EEG from 60 healthy subjects without EEG abnormalities. These 60 data sets were divided into three age groups (20 29, 30 59 and over $60 ; 20$ participants per group) to obtain a rough 


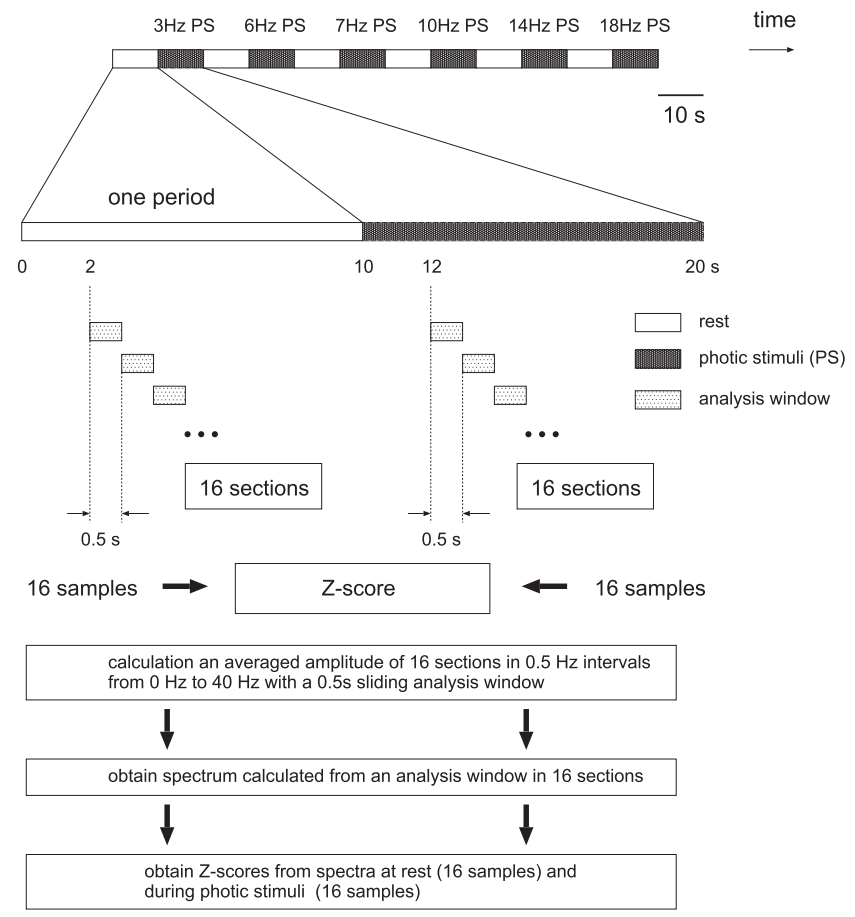

Fig. 1 Flowchart showing method of obtaining Z-score in individual data.

approximation of the relationship between EEG responses and aging. The average age across all 60 participants was $43.9 \pm 19.5$ years. The average ages in each group were $23.7 \pm 1.87,39.8 \pm 7.15$ and $68.5 \pm 6.92$, for the $20 \sim 29$, $30 \sim 59$ and $60+$ years age groups, respectively.

A flowchart of our analysis is shown in Fig. 1. In this study, we defined each section as a block of activity while the participant was either 'at rest' or 'during PS'. Each window shows the shortest data block for analysis, referred to as the analysis window. One sample amplitude value was calculated from each analysis window. These values were used to obtain Z-scores, as described below. EEG measurements at rest and with PS were repeated every $10 \mathrm{~s}$, alternately. In each period, the first $2 \mathrm{~s}$ of data were discarded to avoid the effects of the orienting response, and off-response stimuli. The comparison was thus performed with the remaining $8 \mathrm{~s}$ of data in each block. The photic driving response is not constant over a 10-second period. Rather, the amplitude of all frequency components varies within a short time. For this reason, we divided the EEG waveform into a large number of short segments. We set the window size to cover 100 points (over 0.5 seconds), giving a frequency resolution of $0.5 \mathrm{~Hz}$. Using this window without overlap, we obtained 16 samples from 16 sections to construct a distribution of Z-scores for each analysis frequency, then calculated each PS frequency. In this study, we used a discrete Fourier transform to calculate frequency components. With this method, it is possible to calculate frequency components at integral multiples of the fundamental frequency (i.e. $2 \mathrm{~Hz}$ in this study). However, it is not possible to obtain frequency components with a resolution less than $2 \mathrm{~Hz}$. There- fore, we estimated uncalculated frequency components using the approximation method [6]. The amplitude spectrum was obtained between 0 and $40 \mathrm{~Hz}$, at a resolution of $0.5 \mathrm{~Hz}$. The frequency spectrum can be obtained as the following equation by a trapezoidal integral approximation of the continuous Fourier transform, $X(f)=\int_{-\infty}^{\infty} x(t) e^{-j 2 \pi f t} d t$.

$$
X_{N}^{*}(f)=\Delta t \sum_{k=0}^{N-1} x(k \Delta t) e^{-j 2 \pi f k \Delta t}
$$

where $N$ is the number of sample points in the analysis window. The amplitude spectrum can then be calculated easily from the real and imaginary parts of $X_{N}^{*}(f)$.

We used the Z-scores as an indicator for measuring the distance between two amplitude distributions at rest and during PS. The Z-scores were obtained with Mann-Whitney Utests for comparing pairs of amplitude distributions. This test is based on the Wilcoxon rank-sum test and a nonparametric test for assessing whether two sample sets of observations come from the same distribution. However, samples extracted from consecutive EEG as shown in Fig. 1 may not be independent, because EEG signals are derived from neural activity in the brain, which is not necessarily independent, introducing problems in determining significant differences between two distributions for standard statistical tests. As such, we did not use the Z-score as the threshold for judging whether there were significant intraindividual differences. This means that Z-score in this study is just an indicator for measuring the distance between two distributions.

We defined the rank-sum of two groups consisting of amplitudes at rest and during PS as: $R_{1}$ and $R_{2}\left(R_{1}\right.$ is ahead of $R_{2}$ ) after arranging data in descending rank order for amplitude. We denoted the sample numbers as $N_{1}$ and $N_{2}$, set here to 16 between two sections. U-statistics for the above two distributions are expressed as:

$$
\begin{aligned}
& U_{1}=R_{1}-\frac{N_{1}\left(N_{1}+1\right)}{2} \\
& U_{2}=R_{2}-\frac{N_{2}\left(N_{2}+1\right)}{2}=N_{1} N_{2}+\frac{N_{1}\left(N_{1}+1\right)}{2}-R_{1}
\end{aligned}
$$

The latter equation of Eq. (3) is derived from the relation, $U_{1}+U_{2}=N_{1} N_{2}$. For large samples, the normal distribution approximation can be applied. Finally, the Z-value is calculated by the following equation.

$$
Z=\frac{N_{1} \cdot N_{2} / 2-U_{2}}{\sqrt{N_{1} \cdot N_{2}\left(N_{1}+N_{2}+1\right) / 12}}
$$

\section{Results}

We evaluated amplitudes at the fundamental frequency $\left(f_{0}\right)$, the second harmonic frequency $\left(2 f_{0}\right)$, higher order harmonic frequencies less than $40 \mathrm{~Hz}$, and the alpha frequency. Figure 2 shows the averaged Z-scores at electrode $O_{1}$ elicited by $6 \mathrm{~Hz}$ PS. In addition, the ratio of averaged amplitude at rest and during PS (commonly used as an indicator for 
comparing two subject's states) is presented in Fig. 3. The ratio mentioned above is unstable because the fundamental and higher order harmonics are not clear. In contrast, Fig. 2 shows the calculated Z-scores in our method, reflecting the distance between two amplitude distributions at rest and during $6 \mathrm{~Hz}$ PS. A clear peak in the fundamental frequency $\left(f_{0}\right)$ can be seen, as well as prominent peaks in the harmonic components $\left(n f_{0}, n \geq 2\right)$. The peak at the second harmonic appears to be different from other fundamental and harmonic peaks for the 20 29 years age group, possibly reflecting the effects of alpha suppression due to visual stimulation, since EEG is measured with eyes closed. The Z-scores in alpha frequency band are particularly likely to be affected, because the spectral power is the largest among the rhythmic EEG signals. Considering the potential effects of alpha wave activity, it is appropriate to separate the frequencies included in the alpha frequency band. As such, in this study of enhancement and suppression in response to visual stimuli at fundamental and harmonic frequencies, the alpha frequency band and suppression in the alpha frequency band were examined separately. Here, some harmonics in Figs. 2 and 3 do not have sharp single peaks. We propose two pos-

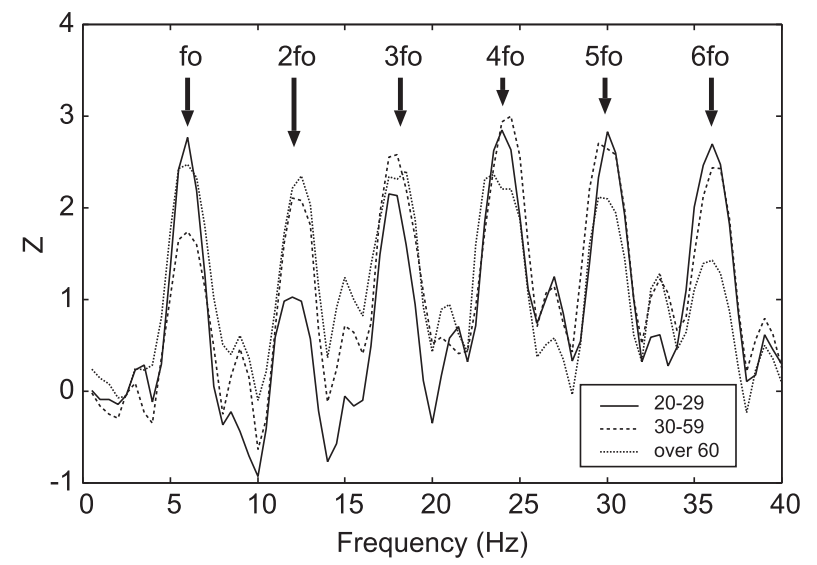

Fig. 2 Example of Z-scores as a function of analysis frequency $(\mathrm{PS} 6 \mathrm{~Hz}$, $\left.O_{1}\right)$.

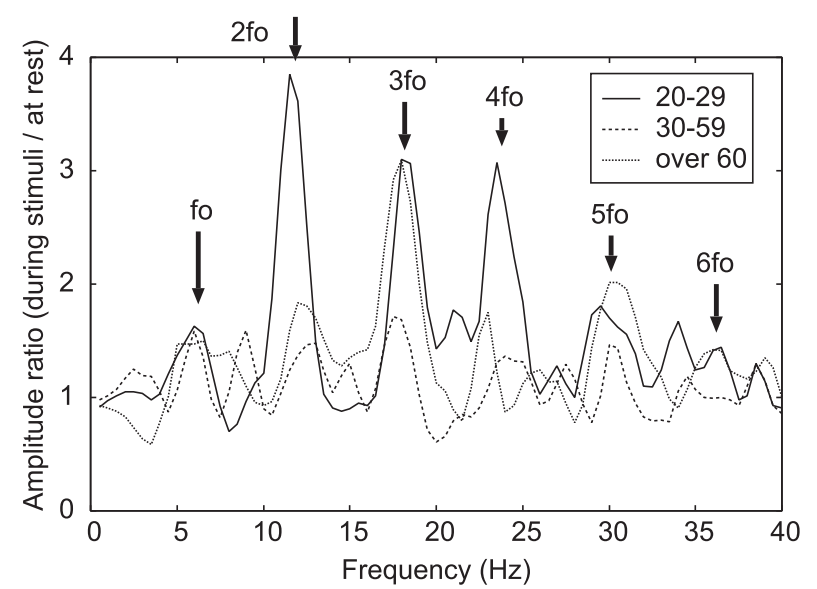

Fig. 3 Amplitude ratio produced by dividing the amplitude during photic stimulation by that at rest (PS $6 \mathrm{~Hz}, O_{1}$ ). sible reasons for this finding. First, there may have been an effect of the approximation by Eq. (1), because frequency resolution was originally $2 \mathrm{~Hz}$. Alternatively, the spreading of the spectrum in the frequency domain may have depended on the length of the analysis window. There is a trade-off between the number of samples used for obtaining Z-scores and the window length, which was chosen, as appropriate, by considering the stability of Z-score.

To examine whether Z-scores acted as an index of aging, we first present the data grouped into three age groups, then examine the relationship between Z-scores and age derived from intraindividual data. The mean and standard deviation of Z-scores are shown for each group with three combinations of parameters for fundamental and harmonic frequencies, and for alpha frequency, in Fig. 4. Here, the combination of parameters was electrode position, PS frequency and harmonic frequency/alpha frequency taken from the parameters showing the smallest $p$-value in statistical tests among three age groups. The asterisk in the figure indicates a significant difference between two age distributions $(p<0.01)$, revealed by Tamhane's multiple comparisons test. Next, we plotted the individual data to obtain Z-scores for aging for the harmonic frequency and alpha frequency (Fig. 5). The function of age (X) and Z-scores (Z) estimated by the method of least squares, and the correlation coefficients $(\mathrm{CC})$ between them are shown in the figure.

\section{Discussion}

Our examination of the two distributions in Z-scores revealed a significant difference, as shown in Fig. 4. Z-scores decreased with aging for harmonic frequency components, while this value increased for alpha frequency. The gradient of the estimated linear function exhibited a negative value for harmonic frequencies and a positive value for the alpha frequency band. The relationship between Z-scores and age shown in Fig. 5 demonstrated a negative correlation with a coefficient of -0.740 . In contrast, the relationship between
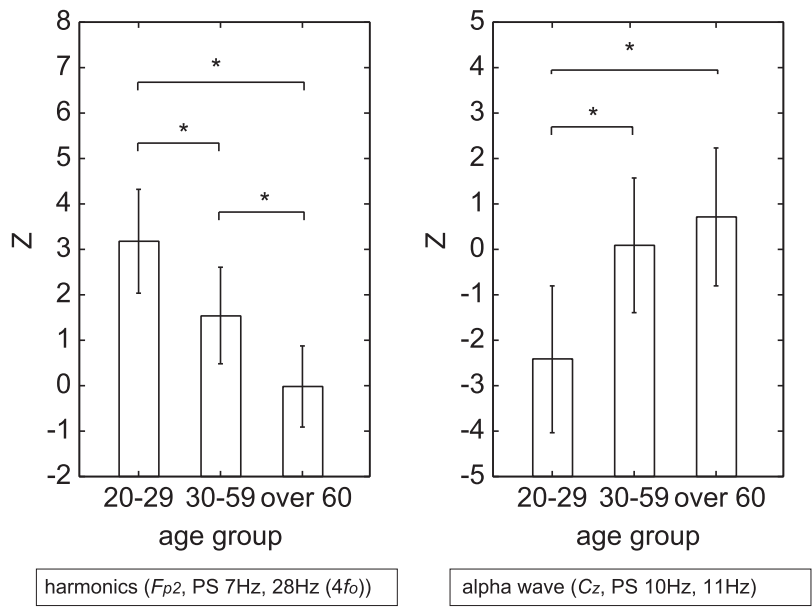

Fig. 4 Average and standard deviation of $Z$-scores in each age group (*: $p<0.01$. 


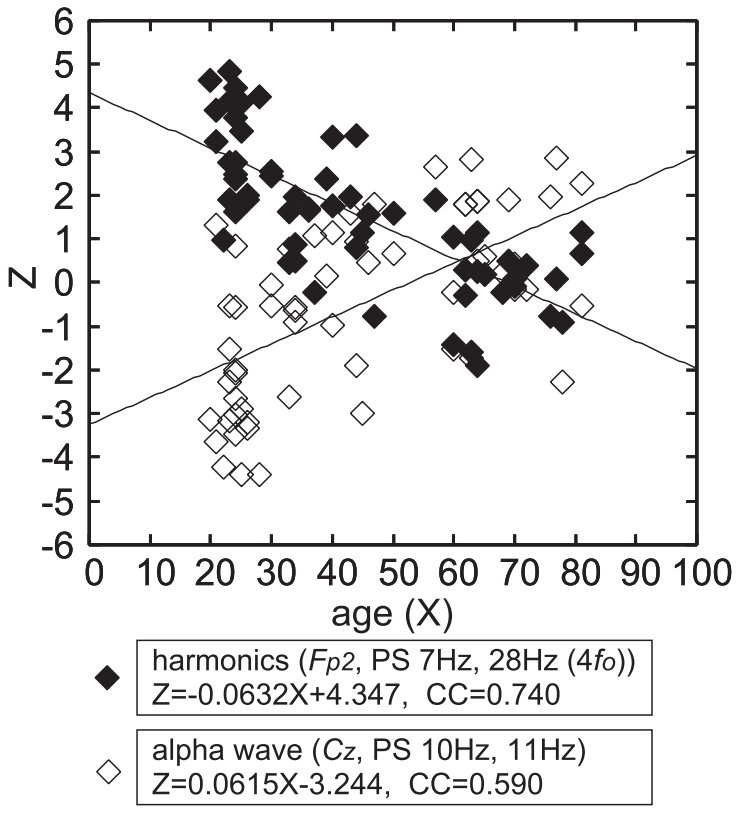

Fig. 5 The relationship between Z-scores and age.

Z-scores and age in the alpha frequency band exhibited a positive correlation, with a coefficient of 0.590 .

We were able to approximate the tendency of Z-score changes with aging using these coefficients. These values were relatively high, especially for harmonic frequencies, though there was substantial variation in the individual data. These results indicate that our novel technique using the photic driving response can provide additional information in conventional routine EEG examinations. This additional information may be helpful in clinical diagnosis.

Regarding electrode position, it has been known that both photic driving response and alpha wave are prominent in the occipital region. However, in this study, the best electrode for identifying aging according to our criterion was $F p_{2}$ for harmonics in the photic driving response, $C_{z}$ for alpha wave.

Because the data in this study were recorded in the course of standard clinical examinations, we were not able to change the EEG measurement conditions to obtain stable
Z-scores and suppress individual variance for fitting linear functions, or improve correlation coefficients. Additional studies involving more samples should be used to construct amplitude distributions to compare between rest and photic stimulation conditions. In future, we plan to test the effect of changing the measurement parameters, such as increasing the measurement period.

\section{Conclusions}

In this study, we examined the possibility of estimating the effects of aging to provide additional information in routine EEG examinations using the photic driving response with a range of PS frequencies. The results revealed that increasing age was associated with a prominent decrease of Z-scores at higher harmonic frequency ranges and an increase in Zscores in the alpha frequency band. We found that Z-scores and age were negatively correlated in the harmonic frequencies but positively correlated in the alpha frequency band. These results suggest that Z-score changes might be used as an index of brain aging, providing additional information for routine EEG examinations. Future research should seek to verify and extend these findings using large sample sizes and improved correlation coefficients between Z-scores and age by reducing individual variance.

\section{References}

[1] D.B. Lindsley, "A longitudinal study of the occipital alpha rhythm in normal children: Frequency and amplitude standards," J. Gen. Psychol., vol.55, pp.197-213, 1939.

[2] E.D. Adrian, "The Berger rhythm: Potential changes from the occipital lobes in man," Brain, vol.57, pp.355-385, 1934.

[3] T. Fukushima, "Application of EEG-interval-spectrum-analysis (EISA) to the study of photic driving responses. A preliminary report," Arch. Psychiatr. Nervenkr., vol.220, pp.99-105, 1975.

[4] de Sá A.M. Miranda and A.F. Infantosi, "Evaluating the entrainment of the alpha rhythm during stroboscopic flash stimulation by means of coherence analysis," Med. Eng. Phys., vol.27, pp.167-173, 2005.

[5] G.G. Celesia, F. Richard, and F. Daly F, "Effects of aging on visual evoked responses," Arch. Neurol., vol.34, pp.403-407, 1977.

[6] B.R. Hunt, "Spectral effects in the use of Newton-cotes approximations for computing discrete Fourier transforms," IEEE Trans. Comput., vol.C-20, no.8, pp.942-943, 1971. 